\title{
Late-epoch optical and near-infrared observations of the GRB 000911 afterglow and its host galaxy ${ }^{\star}$
}

\author{
N. Masetti ${ }^{1}$, E. Palazzi ${ }^{1}$, E. Pian ${ }^{1,2}$, L. Hunt ${ }^{3}$, J. P. U. Fynbo ${ }^{4}$, J. Gorosabel ${ }^{5}$, S. Klose ${ }^{6}$, S. Benetti ${ }^{7}$, R. Falomo ${ }^{7}$, \\ A. Zeh ${ }^{6}$, L. Amati ${ }^{1}$, M. I. Andersen ${ }^{8}$, A. J. Castro-Tirado ${ }^{5}$, J. M. Castro Cerón ${ }^{4,9}$, J. Danziger ${ }^{2}$, F. Frontera ${ }^{1,10}$, \\ A. S. Fruchter ${ }^{9}$, J. Greiner ${ }^{11}$, J. Hjorth ${ }^{4}$, B. L. Jensen ${ }^{4}$, L. Kaper ${ }^{12}$, C. Kouveliotou ${ }^{13}$, A. Levan ${ }^{9}, 14$, A. Magazzù ${ }^{15}$, \\ P. Møller ${ }^{16}$, L. Nicastro ${ }^{17}$, H. Pedersen ${ }^{4}$, N. R. Tanvir ${ }^{18}$, P. M. Vreeswijk ${ }^{19}$, \\ R. A. M. J. Wijers ${ }^{12}$, and E. P. J. van den Heuvel ${ }^{12}$
}

\begin{abstract}
1 INAF - Istituto di Astrofisica Spaziale e Fisica Cosmica, Sezione di Bologna, via Gobetti 101, 40129 Bologna, Italy (formerly IASF/CNR, Bologna) e-mail: masetti@bo.iasf.cnr.it; ${ }^{2}$ INAF - Osservatorio Astronomico di Trieste, via GB Tiepolo 11, 34131 Trieste, Italy; ${ }^{3}$ INAF - Istituto di Radioastronomia, Sezione di Firenze, largo E. Fermi 5, 50125, Florence, Italy; ${ }^{4}$ Niels Bohr Institute, University of Copenhagen, Juliane Maries Vej 30, 2100 Copenhagen Ø, Denmark; ${ }^{5}$ Instituto de Astrofísica de Andalucía (IAA-CSIC), Apartado de Correos 03004, 18080 Granada, Spain; ${ }^{6}$ Thüringer Landessternwarte Tautenburg, 07778 Tautenburg, Germany; ${ }^{7}$ INAF - Osservatorio Astronomico di Padova, vicolo dell'Osservatorio 5, 35122 Padua, Italy; ${ }^{8}$ Astrophysikalisches Institut Potsdam, An der Sternwarte 16, 14482 Potsdam, Germany; 9 Space Telescope Science Institute, 3700 San Martin Drive, Baltimore, MD 21218, USA; ${ }^{10}$ Dipartimento di Fisica, Università di Ferrara, via Paradiso 12, 44100 Ferrara, Italy; ${ }^{11}$ Max-Planck-Institut für Extraterrestrische Physik, Giessenbachstrasse, 85748 Garching, Germany; ${ }^{12}$ Institute of Astronomy "Anton Pannekoek”, University of Amsterdam, Kruislaan 403, 1098 SJ Amsterdam, The Netherlands; ${ }^{13}$ NASA Marshall Space Flight Center, SD-50, Huntsville, AL 35812, USA; ${ }^{14}$ Department of Physics and Astronomy, University of Leicester, University Road, Leicester, LE1 7RH, UK; ${ }^{15}$ INAF - Telescopio Nazionale Galileo, Roque de Los Muchachos Astronomical Observatory, PO Box 565, 38700 Santa Cruz de La Palma, Spain; ${ }^{16}$ European Southern Observatory, Karl Schwarzschild-Strasse 2, 85748 Garching, Germany; ${ }^{17}$ INAF - Istituto di Astrofisica Spaziale e Fisica Cosmica, Sezione di Palermo, via La Malfa 153, 90146 Palermo, Italy (formerly IASF/CNR, Palermo); ${ }^{18}$ Centre for Astrophysics Research, University of Hertfordshire, College Lane, Hatfield, Herts AL10 9AB, UK; ${ }^{19}$ European Southern Observatory, Casilla 19001, Santiago 19, Chile
\end{abstract}

Received 25 January 2005 / Accepted 18 April 2005

\begin{abstract}
We present the results of an optical and near-infrared (NIR) monitoring campaign of the counterpart of Gamma-Ray Burst (GRB) 000911, located at redshift $z=1.06$, from 5 days to more than 13 months after explosion. Our extensive dataset is a factor of 2 larger and spans a time interval $\sim 4$ times longer than the ones considered previously for this GRB afterglow; this allows a more thorough analysis of its light curve and of the GRB host galaxy properties. The afterglow light curves show a single power-law temporal decline, modified at late times by light from a host galaxy with moderate intrinsic extinction, and possibly by an emerging supernova (SN). The afterglow evolution is interpreted within the classical "fireball" scenario as a weakly collimated adiabatic shock propagating in the interstellar medium. The presence of a SN light curve superimposed on the non-thermal afterglow emission is investigated: while in the optical bands no significant contribution to the total light is found from a SN, the NIR $J$-band data show an excess which is consistent with a SN as bright as the known hypernova SN1998bw. If the SN interpretation is true, this would be the farthest GRB-associated SN, as well as the farthest core-collapse SN, discovered to date. However, other possible explanations of this NIR excess are also investigated. Finally, we studied the photometric properties of the host, and found that it is likely to be a slightly reddened, subluminous, extreme starburst compact galaxy, with luminosity $\sim 0.1 L^{\star}$, an age of $\sim 0.5 \mathrm{Gyr}$ and a specific Star Formation Rate $(S F R)$ of $\approx 30 M_{\odot} \mathrm{yr}^{-1}\left(L / L^{\star}\right)^{-1}$. This is the highest specific $S F R$ value for a GRB host inferred from optical/NIR data.
\end{abstract}

Key words. gamma rays: bursts - supernovae: general - radiation mechanisms: non-thermal - cosmology: observations galaxies: high-redshift - Galaxy: fundamental parameters

* Based on observations made with ESO Telescopes at the Paranal and La Silla Observatories under programmes 165.H-0464 and 265.D-5742, with the Italian Telescopio Nazionale Galileo and with the Nordic Optical Telescope (NOT), both operating on the island of La Palma in the Spanish Observatorio del Roque de los Muchachos of

the Instituto de Astrofísica de Canarias. The NOT is operated jointly by Denmark, Finland, Iceland, Norway, and Sweden. 


\section{Introduction}

Long and frequent ground-based monitoring at optical and near-infrared (NIR) wavelengths of Gamma-Ray Burst (GRB) counterparts is necessary to constrain the emission models and disentangle the contribution of the GRB host galaxy and of a possible underlying supernova ( $\mathrm{SN}$ ) explosion simultaneous with or shortly preceding the GRB (e.g., McFadyen \& Woosley 1999; Vietri \& Stella 1998).

The connection of GRBs with $\mathrm{SNe}$ was first suggested by the close angular and temporal proximity of GRB 980425 and SN1998bw (Galama et al. 1998) and, based on that event, this association was systematically explored (e.g., Castro-Tirado \& Gorosabel 1999; Bloom et al. 1999: Galama et al. 2000; Reichart 2001; Garnavich et al. 2003; Greiner et al. 2003; Price et al. 2003a; Masetti et al. 2003). The recent direct spectroscopic detections of SNe 2002lt (Della Valle et al. 2003), 2003dh (Stanek et al. 2003; Hjorth et al. 2003) and 20031w (Malesani et al. 2004) associated with GRBs 021211, 030329 and 031203, respectively, firmly established that at least a fraction of GRBs is connected with core-collapse SNe.

However, given the high redshifts $(z \gtrsim 1)$ of most GRBs, $\mathrm{SNe}$ associated with them are difficult to study spectroscopically, and only good observational coverage of the optical light curve can reveal the presence of an underlying SN (see Zeh et al. 2004; Dar \& De Rújula 2003). In addition, due to the possible dust obscuration near the source, NIR data may play an even more important role than optical data (e.g., Palazzi et al. 1998; Gorosabel et al. 1998; Pian et al. 1998; Vreeswijk et al. 1999; Castro-Tirado et al. 1999; Masetti et al. 2000; Klose et al. 2000; Rhoads \& Fruchter 2001; Sokolov 2001; Le Floc'h et al. 2003). Thus, indirect (i.e. late-epoch photometric) observations of the presence of a $\mathrm{SN}$ are still important in the study of the GRB-SN connection.

GRB 000911 was detected by the Inter-Planetary Network (IPN) satellites on 2000 September 11.30237 UT as a very long ( 500 s) burst (Hurley et al. 2000; Price et al. 2002). Shortly thereafter, Berger et al. (2000) found a variable radio source inside the GRB error box and, at the radio position, Price et al. (2000, 2002) detected an optical transient (OT) at coordinates RA $=02^{\mathrm{h}} 18^{\mathrm{m}} 34^{\mathrm{s}} .36$, Dec $=+07^{\circ} 44^{\prime} 27^{\prime \prime} .65(\mathrm{~J} 2000)$ which faded according to a power-law decay $F(t) \propto t^{-\alpha}$ with index $\alpha=1.46$. This behaviour, together with the positional coincidence with the mentioned transient radio source, strongly suggested that this OT was indeed the optical afterglow of GRB 000911.

Smith et al. (2001) reported no detection of transient emission at sub-mm $(850 \mu \mathrm{m})$ wavelengths. In this same band, Berger et al. (2003) reported the detection of emission from the host galaxy of GRB 000911; this detection was however questioned by Tanvir et al. (2004) on the basis of the former sub-mm upper limit of Smith et al. (2001) for the combined host plus afterglow emission. Extensive optical and NIR observations of the afterglow have been reported by Lazzati et al. (2001), who inferred the presence of a $\mathrm{SN}$ reaching maximum light at about 18 days after the GRB (in the rest frame at $z=1.0585$ : Djorgovski et al. 2001; Price et al. 2002). The underlying host galaxy appears to be a starburst with moderate dust absorption, i.e. with $0.11<E(B-V)<0.21$ (Lazzati et al. 2001). No follow-up observations of this GRB were performed in X-rays.

As part of our ESO program, in the framework of the GRACE $^{1}$ collaboration, we observed the field of GRB 000911 with both optical and NIR cameras. Our observations started as soon as the precise position of the radio counterpart was made public; we then followed up the optical/NIR afterglow for more than one year with various telescopes located at ESO and at the Canary Islands Observatories. In this paper we present our data and compare the results with those published in Lazzati et al. (2001). In particular, since our dataset is more complete in terms of time coverage and contains twice as many measurements as reported by those authors, we have a chance to test with higher confidence the presence of a $\mathrm{SN}$. Moreover, our late-time observations allowed us to study in detail the broadband optical/NIR emission from the host galaxy of GRB 000911.

Throughout this paper we will assume a cosmology with $H_{0}=65 \mathrm{~km} \mathrm{~s}^{-1} \mathrm{Mpc}^{-1}, \Omega_{\Lambda}=0.7, \Omega_{\mathrm{m}}=0.3$. Throughout the text, unless otherwise stated, errors and upper limits are at $1 \sigma$ and $3 \sigma$ confidence level, respectively.

\section{Observations and data reduction}

We started our observational campaign on the OT of GRB 000911 at ESO on September 16, 2000. The complete $\log$ of our imaging observations is reported in Table 1.

\subsection{Optical photometry}

Optical BVRI data were collected at Cerro Paranal (Chile) with VLT-Antu plus FORS1 and with VLT-Kueyen plus FORS2 over a baseline of more than 13 months. FORS1 and FORS2 were equipped with a Tektronix and a SiTE CCD, respectively, in both cases with a $2048 \times 2048$ pixel array; both instruments covered a 6 ' $^{\prime} \times 6$ ' $^{\prime} 8$ field in the standard resolution imaging mode, giving a scale of $0.2 \mathrm{pix}^{-1}$.

A single $B$-band pointing was obtained on September 23, 2000, with TNG+DOLoRes at La Palma, Canary Islands (Spain); the imaging spectrograph DOLoRes carried a $2048 \times 2048$ pixel Loral backside-illuminated CCD which images a field of $9.5 \times 9.5$ with a scale of $0.275 \mathrm{pix}^{-1}$. Early $I$-band and late-time $R$ - and $I$-band observations were acquired at NOT (La Palma, Canary Islands), with the ALFOSC instrument. This also was equipped with a $2048 \times 2048$ pixel Loral CCD, giving a field of view of $6.4 \times 6.4$ and an image scale of $0{ }^{\prime} 188 \mathrm{pix}^{-1}$.

Optical images were bias-subtracted and flat-fielded with the standard reduction procedure. In general, frames taken on the same night in the same band were summed together in order to increase the signal-to-noise ratio. We performed photometry with standard Point Spread Function (PSF) fitting using the DAOPHOT II image data analysis package PSF-fitting

1 GRB Afterglow Collaboration at ESO: see the web page http: //www.gammaraybursts.org/grace/ 
Table 1. Journal of the optical and NIR photometry of the GRB 000911 afterglow. Magnitude uncertainties are at $1 \sigma$ confidence level; upper limits are at $3 \sigma$ confidence level. The magnitudes are not corrected for Galactic interstellar extinction.

\begin{tabular}{|c|c|c|c|c|c|}
\hline $\begin{array}{c}\text { Mid-exposure } \\
\text { time (UT) }\end{array}$ & Telescope & Filter & $\begin{array}{c}\text { Total exposure } \\
\text { time (s) }\end{array}$ & $\begin{array}{l}\text { Seeing } \\
(\operatorname{arcsec})\end{array}$ & Magnitude \\
\hline 2000 Sep. 16.328 & Kueyen & $B$ & $6 \times 1200$ & 0.8 & $23.3 \pm 0.3^{*}$ \\
\hline 2000 Sep. 23.075 & TNG & $B$ & $3 \times 600$ & 1.1 & $24.77 \pm 0.10$ \\
\hline 2000 Sep. 28.326 & Antu & $B$ & $5 \times 180$ & 1.0 & $24.91 \pm 0.12$ \\
\hline 2000 Dec. 22.163 & Antu & $B$ & $4 \times 300$ & 0.8 & $>25.5$ \\
\hline 2000 Sep. 16.328 & Kueyen & $V$ & $6 \times 1200$ & 0.8 & $22.9 \pm 0.2^{*}$ \\
\hline 2000 Sep. 19.288 & Antu & $V$ & $5 \times 180$ & 0.7 & $23.66 \pm 0.07$ \\
\hline 2000 Sep. 21.357 & Antu & $V$ & $7 \times 180$ & 0.7 & $23.87 \pm 0.07$ \\
\hline 2000 Sep. 28.339 & Antu & $V$ & $5 \times 180$ & 1.0 & $24.45 \pm 0.1$ \\
\hline 2000 Dec. 22.181 & Antu & V & $3 \times 300$ & 0.9 & $25.43 \pm 0.3$ \\
\hline 2001 Oct. 25.203 & Antu & $V$ & $4 \times 300$ & 0.6 & $25.35 \pm 0.3$ \\
\hline 2000 Sep. 16.235 & Kueyen & $R$ & $2 \times 60$ & 0.8 & $22.42 \pm 0.15$ \\
\hline 2000 Sep. 19.274 & Antu & $R$ & $5 \times 180$ & 0.6 & $23.12 \pm 0.05$ \\
\hline 2000 Sep. 21.374 & Antu & $R$ & $5 \times 180$ & 0.8 & $23.42 \pm 0.05$ \\
\hline 2000 Sep. 28.313 & Antu & $R$ & $5 \times 180$ & 0.8 & $24.01 \pm 0.08$ \\
\hline 2000 Sep. 30.244 & Antu & $R$ & $4 \times 60$ & 0.8 & $24.12 \pm 0.10$ \\
\hline 2000 Dec. 22.194 & Antu & $R$ & $3 \times 300$ & 1.0 & $>24.2$ \\
\hline 2001 Aug. 15.659 & NOT & $R$ & $15 \times 900$ & 1.1 & $25.27 \pm 0.15$ \\
\hline 2000 Sep. 15.097 & NOT & $I$ & $4 \times 420$ & 1.4 & $21.05 \pm 0.3$ \\
\hline 2000 Sep. 16.328 & Kueyen & $I$ & $6 \times 1200$ & 0.8 & $21.9 \pm 0.2^{*}$ \\
\hline 2000 Sep. 18.333 & Antu & $I$ & $1 \times 180$ & 0.9 & $22.11 \pm 0.10$ \\
\hline 2000 Sep. 19.261 & Antu & $I$ & $5 \times 180$ & 0.7 & $22.55 \pm 0.06$ \\
\hline 2000 Sep. 21.388 & Antu & $I$ & $5 \times 180$ & 0.8 & $22.78 \pm 0.07$ \\
\hline 2000 Sep. 28.353 & Antu & $I$ & $5 \times 180$ & 0.9 & $23.35 \pm 0.15$ \\
\hline 2000 Oct. 26.177 & Antu & $I$ & $5 \times 180$ & 0.7 & $23.93 \pm 0.15$ \\
\hline 2000 Dec. 22.208 & Antu & $I$ & $3 \times 300$ & 1.1 & $>23.0$ \\
\hline 2001 Aug. 14.166 & NOT & $I$ & $15 \times 600$ & 0.9 & $24.3 \pm 0.2$ \\
\hline 2001 Sep. 17.377 & Antu & $I$ & $3 \times 360$ & 0.9 & $24.25 \pm 0.15$ \\
\hline 2001 Sep. 18.399 & Antu & $I$ & $3 \times 360$ & 0.8 & $24.45 \pm 0.25$ \\
\hline 2001 Sep. 24.375 & Antu & $I$ & $3 \times 360$ & 0.6 & $23.78 \pm 0.17$ \\
\hline 2000 Sep. 22.230 & NTT & $J$ & 900 & 0.9 & $22.0 \pm 0.2$ \\
\hline 2000 Dec. 22.125 & Antu & $J$ & 1800 & 0.6 & $>23.4$ \\
\hline 2000 Sep. 22.240 & NTT & $H$ & 900 & 0.9 & $21.3 \pm 0.2$ \\
\hline 2000 Sep 28.200 & NTT & $H$ & 3240 & 1.3 & $>22.1$ \\
\hline 2000 Dec. 22.063 & Antu & $H$ & 5400 & 0.6 & $22.6 \pm 0.35$ \\
\hline 2000 Sep. 22.250 & NTT & $K_{\mathrm{s}}$ & 900 & 0.8 & $19.9 \pm 0.1$ \\
\hline
\end{tabular}

*This measurement was derived from a spectroscopic observation.

algorithm (Stetson 1987) within MIDAS ${ }^{2}$. The PSF-fitting photometry is accomplished by modeling a two-dimensional Gaussian profile with two free parameters (the half width at

\footnotetext{
${ }^{2}$ MIDAS (Munich Image Data Analysis System) is developed, distributed and maintained by ESO (European Southern Observatory) and is available at http://www.eso.org/projects/esomidas
}

half maxima along $x$ and $y$ coordinates of each frame) on at least 5 unsaturated bright stars in each image. The errors associated with the measurements reported in Table 1 represent statistical uncertainties obtained with the standard PSF-fitting procedure.

For the late-epoch observations (i.e. those taken starting December 2000), when the OT was faint and a substantial 
contribution from an underlying host galaxy became apparent (see Sect. 3.1), we checked the results of the PSF-fitting algorithm by determining the optical magnitudes of the object with the aperture photometry method. In this case we used an aperture diameter equal to the Full Width at Half Maximum ( $F W H M$ ) of each summed image. Comparison between the results obtained with the two methods indicates no appreciable difference within the uncertainties. As we shall see below, this has implications for the compactness and the morphology of the host of GRB 000911.

To be consistent with optical magnitude measurements appearing on the GCN circulars archive ${ }^{3}$, the photometric calibration was done using the $B V R I$ magnitudes, as measured by Henden (2000), of several field stars checked for their magnitude constancy. We find this magnitude calibration to be accurate to within $3 \%$. However, this calibration is offset with respect to the one used by Lazzati et al. (2001), depending on the considered band, by $\sim 0.1-0.2 \mathrm{mag}$. We have evaluated this discrepancy by comparing our and their quasi-simultaneous $(<2 \mathrm{~h})$ measurements of September 28, 2000, and have corrected their photometry to obtain a mutually consistent zero-point level. Because of the above procedure, we did not plot in Fig. 2 the measurements acquired by Lazzati et al. (2001) on that date. It should also be noted that the photometry errors quoted throughout the rest of the paper are only statistical and do not account for any possible zero-point offset, which we expect to be smaller than $2 \%$.

\subsection{NIR photometry}

NIR imaging in $J, H$ and $K_{\mathrm{s}}$ bands was obtained between September 21 and December 22, 2000, at the ESO NTT+SofI in La Silla (Chile), and again at the VLT-Antu in Paranal (Chile), equipped with ISAAC (see Table 1). The SofI NIR camera carried a Rockwell Hawaii $1024 \times 1024$ pixel $\mathrm{HgCdTe}$ array for imaging and spectroscopy in the $0.9-2.5 \mu \mathrm{m}$ band. The plate scale was 0.292 pix $^{-1}$ and the corresponding field of view was $4.9 \times 4 ! 9$. ISAAC was equipped, in the shortwavelength $(0.9-2.5 \mu \mathrm{m})$ NIR range, with a similar Rockwell Hawaii $1024 \times 1024$ pixel $\mathrm{HgCdTe}$ array which had a scale of $0 !^{\prime} 148 \mathrm{pix}^{-1}$ and a field of view of $2.5 \times 2.5$. The $K_{\mathrm{s}}$ filter is centered at $2.12 \mu \mathrm{m}$ and has a $F W H M$ of $0.34 \mu \mathrm{m}$. For each NIR observation the total integration time was split into images of $40 \mathrm{~s}$ each, with dithering after each individual exposure.

Reduction of the NIR images was performed with the IRAF and STSDAS packages ${ }^{4}$. Each image was reduced by first subtracting a mean sky, obtained from the median of a number of frames acquired just before and after each processed image.

\footnotetext{
${ }^{3}$ GCN Circulars are available at: http://gcn.gsfc.nasa.gov/gcn/gcn3_archive.html

${ }^{4}$ IRAF is the Image Analysis and Reduction Facility made available to the astronomical community by the National Optical Astronomy Observatories, which are operated by AURA, Inc., under contract with the US National Science Foundation. STSDAS is distributed by the Space Telescope Science Institute, which is operated by the Association of Universities for Research in Astronomy (AURA), Inc., under NASA contract NAS 5-26555. It is available at http://iraf.noao.edu/
}

Before frames were used for sky subtraction, stars in them were eliminated by a background interpolation algorithm (imedit) combined with an automatic "star finder" (daofind). Then, a differential dome flatfield correction was applied to the skysubtracted image, and the frames were registered to fractional pixels and combined. The telescope dithering was measured from the offsets of field objects in each image and the images were averaged together using inter-pixel shifts. Magnitudes were measured inside circular apertures of diameter comparable to the $F W H M$ of each image.

We calibrated the NIR photometry with stars selected from the NICMOS Standards List (Persson et al. 1998). The stars were observed in five positions on the detector, and were reduced in the same way as the source observations. Formal photometric accuracy based only on the standard star observations is typically better than $3 \%$. We checked the consistency of our $J$-band calibration with that determined by Lazzati et al. (2001): we found that the two zero points agree well within the uncertainties.

\subsection{Optical spectroscopy}

Two series of 6 spectra each, with individual exposure times of $20 \mathrm{~min}$, were acquired on September 16.328 and 30.301, 2000 (UT times at mid-exposure), with VLT-Kueyen+FORS2 and VLT-Antu+FORS1, respectively. The spectra were acquired using FORS Grism \#150I, which nominally covers the 3300-9000 ̊ range, and with a slit width of $1^{\prime \prime}$. The use of a separation filter was needed to avoid overlapping of spectral orders over a given wavelength; this reduced the spectral range of the two spectra to $3800-8600 \AA$. This setup secured a final dispersion of $5.5 \AA \mathrm{pix}^{-1}$ for the two spectra.

The spectra, after correction for flat-field and bias, were background subtracted and optimally extracted (Horne 1986) using IRAF. Helium-Neon-Argon lamps were used for wavelength calibration; both spectra were then flux-calibrated by using the spectroscopic standard EG21 (Hamuy et al. 1992, 1994). Finally, spectra taken within the same observing run were averaged together to increase the $\mathrm{S} / \mathrm{N}$ ratio. The accuracy of the wavelength and flux calibrations was checked against the position of night sky lines and the photometric data collected around the epoch in which the spectra were acquired, respectively. The typical error was $0.5 \AA$ for the wavelength calibration and $10 \%$ for the flux calibration. Unfortunately, the proximity of the Moon to the OT in the first spectroscopic run and the faintness of the OT in the second one severely limited the quality of these spectra. Thus, no significant spectral features were found, but we were able to extract rough estimates of broadband BVI magnitudes (see Table 1).

\section{Results}

\subsection{GRB 000911 optical-NIR afterglow light curves}

In Fig. 1 a VLT image of the GRB 000911 field is shown. The OT variability and the consistency of its position with both the IPN error box and the radio transient support the afterglow nature of the source indicated by the tick marks. In this deep 

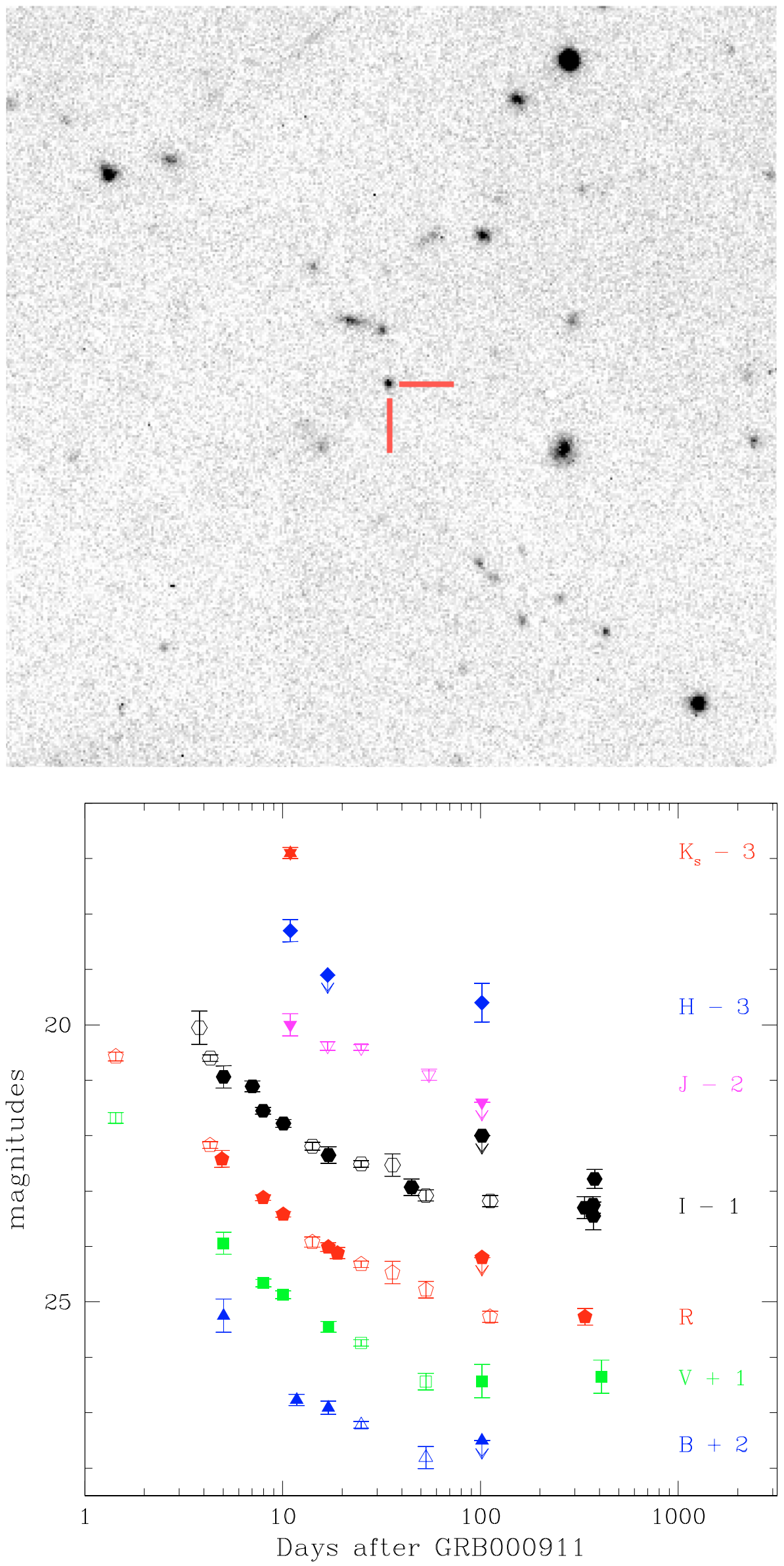

Fig. 1. VLT-Antu $R$-band image of the afterglow of GRB 000911 acquired on September 19, 2000. The field size is about $1^{\prime} .2 \times 1.2$; North is at top, East is to the left. The OT is indicated by the tick marks, and several faint extended objects can be seen around it. The limiting magnitude of the image is $R \sim 25.5$.

Fig. 2. Light curves of the afterglow of GRB 000911. Filled symbols represent our data, open symbols refer to the data reported in Lazzati et al. (2001) and Price et al. (2002). Different symbols correspond to different optical/NIR filters. The data are not corrected for Galactic extinction. 
image (limiting magnitude: $R \sim 25.5$ ), several faint extended objects can be seen around the OT. In Fig. 2 we plot both our photometric measurements and those presented in Lazzati et al. (2001), plus two late $R$ and $I$ points ${ }^{5}$ obtained in January 2001 by Price et al. (2002). In the optical bands, the initial decline slows down at about 20 days after GRB, probably due to the underlying host galaxy, or possibly to the presence of a supernova, as proposed by Lazzati et al. (2001). The NIR light curves are not well sampled, but the $J$ and $H$ band observations also show a fading. We also note (see Fig. 2 and Table 1) that at late times, $\sim 12$ months after the GRB, the I-band light curve shows a marginal increase of $\sim 0.6 \mathrm{mag}$ in 1 week. Due to this behaviour, we excluded the last $I$ point from our light curve fits described below. Possible interpretations of this behaviour are briefly discussed in Sect. 4.1. The $R$ and $I$ observations acquired with VLT-Antu on December 22, 2000 ( $\sim 100$ days after the GRB trigger) were performed under relatively poor seeing conditions and only provide loose upper limits to the OT magnitudes.

We evaluated the Galactic extinction in the optical and NIR bands in the direction of GRB 000911 using the Galactic dust infrared maps by Schlegel et al. (1998); from these data we obtained a color excess $E(B-V)=0.120$. By applying the relation of Cardelli et al. (1989), we derived $A_{B}=0.51$, $A_{V}=0.38, A_{R}=0.32, A_{I}=0.23, A_{J}=0.13, A_{H}=0.07$ and $A_{K_{\mathrm{s}}}=0.04$. The corrected magnitudes were then converted into flux densities following Fukugita et al. (1995) for the optical filters and Bersanelli et al. (1991) for the NIR ones. We also added a $5 \%$ error in quadrature to the uncertainties on the optical-NIR flux densities in order to account for differences in the instrumental responses of different telescopes in the same band.

The optical and NIR colors of the OT of GRB 000911 during the first 10 days after the high-energy event fall in the loci populated by GRB afterglows in the color-color diagrams as illustrated by Šimon et al. (2001) and by Gorosabel et al. (2002).

\subsection{The GRB 000911 host galaxy}

The host galaxy of GRB 000911 appears unresolved in all of our VLT frames, as the PSF of the galaxy image is always consistent with that of a pointlike object. This implies an upper limit of $3.3 \mathrm{kpc}$ on the host galaxy half-light radius. To estimate the flux of the GRB 000911 host, we considered the measurements or upper limits acquired since December 2000 as reported in Table 1 . In case of detections, when more than one measurement was available in the same band, we considered their average value.

The fit to the GRB 000911 host galaxy Spectral Flux Distribution (SFD) was carried out with the use of the version 1.1 of the Hyperz ${ }^{6}$ code (Bolzonella et al. 2000a). This allows a $\chi^{2}$ fit of the observed data with 8 different templates of synthetic galaxy spectra (starburst, elliptical, lenticular, four

\footnotetext{
5 These two points, having been calibrated following Lazzati et al. (2001), were also corrected for the zero-point offset described in Sect. 2.1.

${ }^{6}$ Available at: http://webast .ast.obs-mip.fr/hyperz/
}

kinds of spirals, and irregular). In all cases, the evolution of the Star Formation Rate (SFR) in the galaxy templates is modeled using an exponential law. The Initial Mass Function is modeled assuming the law by Miller \& Scalo (1979). In the fits a solar metallicity $Z=Z_{\odot} \simeq 0.02$ (with $Z$ the mass fraction of heavy elements) was assumed.

This code also allows an estimate of the photometric redshift of the studied galaxy, the age of its dominant stellar population, and the presence of further overall absorption local to the galaxy itself. Four possible extinction laws were considered, i.e., those by Seaton (1979), Fitzpatrick (1986), Prévot et al. (1984) and Calzetti et al. (2000) which are suitable to describe the extinctions within the Milky Way, the Large Magellanic Cloud (LMC), the Small Magellanic Cloud (SMC) and a generic starburst (Stb) galaxy, respectively.

By considering the templates over a redshift range $z=0-5$ with a step $\Delta z=0.001$, and varying the $V$-band local extinction $A_{V}$ in the range $0-2$ with $\Delta A_{V}=0.01$, we find that the template which best fits our VLT VRIH data points and $B J$ upper limits of the GRB 000911 host is that of an irregular galaxy at $z=0.904$ with the presence of slight reddening due to either a LMC-, or a SMC-, or a Stb-like absorption law (see Table 2 and Fig. 3). The spectroscopically determined redshift $(z=1.0585 \pm 0.0001$; Price et al. 2002) is within the $68 \%$ confidence level interval of that obtained from our photometry using the Hyperz code (see Table 2). The best-fit age of the host galaxy stellar population is $0.7 \mathrm{Gyr}$ for a SMC-like template and $0.5 \mathrm{Gyr}$ for LMC- and Stb-like templates. Extinction is moderate with $A_{V}=0.3-0.5$.

The assumption of an evolving metallicity, as remarked by several authors (e.g., Bolzonella et al. 2000a,b; Gorosabel et al. 2003a,b; Christensen et al. 2004), can be considered as a second-order parameter of the fit; thus, this is not expected to significantly alter our results and conclusions. The same applies to the choice of a LMC- or a SMC-like template, instead of a starburst, for the host galaxy.

Using the best-fit galaxy template we have computed the expected host flux in the $B, J$ and $K_{\mathrm{s}}$ band, for which we only have either upper limits or no late-time observations. The magnitudes, not corrected for Galactic extinction, are $B=26.1$, $J=23.6$ and $K_{\mathrm{s}}=21.9$. For each of these three values an uncertainty of 0.3 mag can be assumed.

\subsection{GRB 000911 afterglow light curve fits}

In order to study the GRB 000911 optical/NIR afterglow light curves and to determine the presence of an underlying SN contribution, we took into account the host galaxy contamination as measured at late epochs with VLT (VRIH bands) or as inferred through the Hyperz fits (BJK $K_{\mathrm{s}}$ bands). The host magnitudes and upper limits were also corrected for Galactic reddening and converted to flux densities in the same way applied to the afterglow magnitudes as described in Sect. 3.1.

We then fitted each of the BVRIJ light curves with a powerlaw plus a constant (the latter simulating the galaxy contribution). The results, reported in the upper part of Table 3, show that throughout the optical range, the decay slope is consistent 
Table 2. Best-fit parameters of the GRB 000911 host galaxy obtained with the Hyperz code. Photometric redshift errors are at $68 \%$ confidence level. The value of the luminosity $L^{\star}$ at the knee of the galaxies luminosity distribution (as defined by Schechter 1976; see Sect. 4.3) is taken from Lilly et al. (1995).

\begin{tabular}{ccccccc}
\hline \hline $\begin{array}{c}\text { Template } \\
\text { galaxy }\end{array}$ & $\chi^{2}$ /d.o.f. & $\begin{array}{c}\text { Photometric } \\
\text { redshift }\end{array}$ & $\begin{array}{c}\text { Age } \\
(\mathrm{Gyr})\end{array}$ & $\begin{array}{c}A_{\mathrm{V}} \\
(\mathrm{mag})\end{array}$ & $M_{\mathrm{B}}$ & $L / L^{\star}$ \\
\hline Irr-LMC & 0.685 & $0.904_{-0.128}^{+0.125}$ & 0.509 & 0.39 & -18.40 & 0.066 \\
Irr-Stb & 0.731 & $0.904_{-0.130}^{+0.134}$ & 0.509 & 0.55 & -18.38 & 0.066 \\
Irr-SMC & 0.732 & $0.904_{-0.138}^{+0.149}$ & 0.719 & 0.32 & -18.37 & 0.064 \\
\hline
\end{tabular}

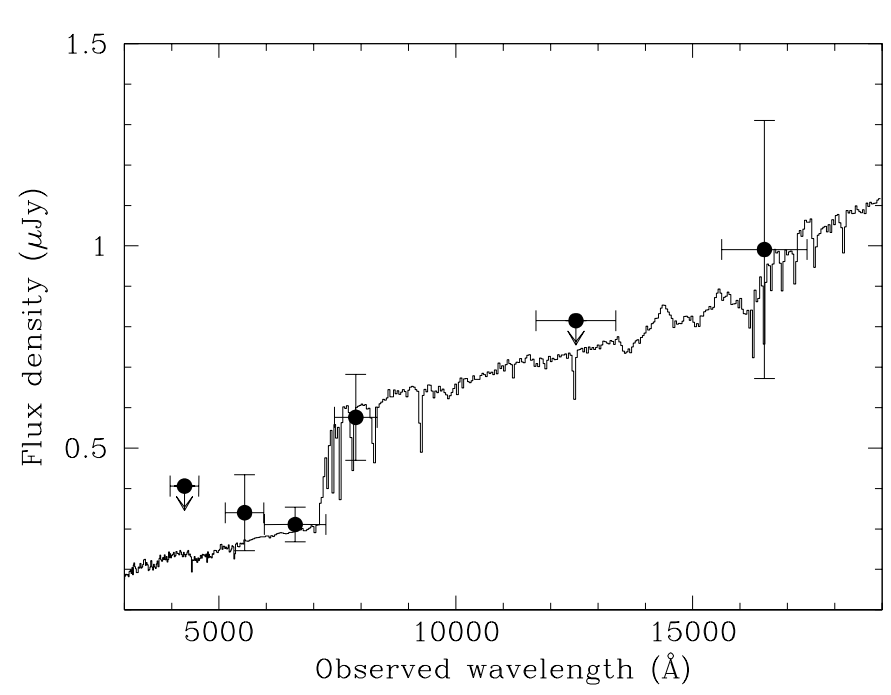

Fig. 3. BVRIJH SFD of the host galaxy of GRB 000911 corrected for Galactic extinction (filled dots), superimposed onto one of the bestfit templates of Table 2 (a starburst-like galaxy plus intrinsic reddening) obtained applying the Hyperz code to the SFD data of the GRB 000911 host. The horizontal bars associated with the SFD data points indicate the $F W H M$ of the photometric filters.

with a constant value. As one can see from the $\chi^{2}$ values, the optical light curves are well described by this model with no need for any further component. The $J$-band data instead appear to have a substantially flatter decay $\left(\alpha_{J}=0.79 \pm 0.15\right)$ and an unacceptable fit $\left(\chi^{2} /\right.$ d.o.f. $=2.6$, where "d.o.f." stands for "degrees of freedom").

However, given that the $J$-band light curve is not sampled in the first 10 days after the GRB, we adopted the same decay slope for the BVRIJ bands assuming the weighted average decay index found in the optical bands: this resulted in $\alpha_{\text {opt }}=1.48 \pm 0.02$. The fit results, illustrated in the central part of Table 3 and in Fig. 4, are again basically acceptable for the optical light curves, except for the $J$-band. Therefore, following Lazzati et al. (2001), we added the prototypical "hypernova" SN1998bw (Galama et al. 1998; Patat et al. 2000) redshifted to $z=1.0585$ (Djorgovski et al. 2001; Price et al. 2002) and dimmed accordingly; while fitting the light curve in each band, we also left the SN flux normalization vary as a free parameter. Details on this procedure can be found, e.g., in Appendix A of Zeh et al. (2004).

Our approach is more general than that of Lazzati et al. (2001); while those authors consider a collimated post-break fireball evolution which requires that the decay index be twice the spectral slope (as in Sari et al. 1999), we do not make any a priori assumption about the fireball hydrodynamics. We also have not tried to estimate a possible supernova contribution to the $B$ and $V$ observed light curves, because there are no ultraviolet (UV) data for SN1998bw to be used as templates to be transformed to those observed bands. Finally, we chose not to simulate UV light curves via extrapolation of the optical spectrum owing to the unpredictably faster variability of the UV flux with respect to the optical flux in supernovae (Cappellaro et al. 1995).

The lower part of Table 3 shows that this model produces a good description of the RIJ dataset (see Fig. 5). The fit improvement obtained by including the presence of a $\mathrm{SN}$, as measured by applying the $F$-test procedure (e.g., Press et al. 1992), is real at a confidence level of $92 \%$. The difference between the results by Lazzati et al. (2001) and the present ones can be explained partly by our much more accurate estimate of the host galaxy $J$-band magnitude, and partly by the fact that those authors did not fully take into account the limitations in the applicability of the $F$-test (see Protassov et al. 2002), namely, the interdependence among fit parameters. Indeed, considering these limitations, one obtains an actual significance of $\sim 85 \%$ for the presence of a SN in the GRB 000911 light curves presented by Lazzati et al. (2001).

Our fits are thus consistent with a SN at a flux comparable to that of SN 1998bw, having exploded simultaneously (with an uncertainty of about one week) with GRB 000911, thus confirming the findings of Lazzati et al. (2001). Possibly, a slight preference (though not statistically significant) for the SN having exploded $\sim 1$ week before the GRB is implied by the $J$-band fits. We remark that the fits reported in the lower part of Table 3 and illustrated in Fig. 5 are made assuming simultaneity between the GRB and SN explosions.

We also attempted fits with different type Ia and Ic SN templates, chosen among those which have well-sampled light curves from the earliest epochs. Specifically, we used SNe 2002ap (Gal-Yam et al. 2002; Pandey et al. 2003), 1994D and 1994I (Barbon et al. 1999, and references therein) and obtain, in terms of statistical confidence, results similar to those derived with SN1998bw.

The $H$-band light curve has not been fitted, because of insufficient sampling. The $H$-band upper limit would suggest a much steeper decay than observed in the other bands. Although we have no reason to believe that the upper limit is not reliable, owing to the scarcity of measurements in this band we do not speculate about this faster decay. 
Table 3. Best-fit parameters of the GRB 000911 afterglow light curve in the BVRIJ bands, computed assuming a power law decay plus a constant (host galaxy) contribution with and without the presence of an underlying 1998bw-like SN occurring simultaneously with the GRB. Host galaxy magnitudes reported here are not corrected for Galactic reddening. Values within brackets are fixed in the corresponding fit.

\begin{tabular}{ccccc}
\hline \hline Filter & $\alpha$ & Host galaxy magnitude & SN intensity ${ }^{a}$ & $\chi^{2} /$ d.o.f. \\
\hline \multicolumn{5}{c}{ Fits without SN plus free $\alpha$} \\
\hline$B$ & $1.12 \pm 0.17$ & {$[26.1]$} & - & $4.2 / 4$ \\
$V$ & $1.63 \pm 0.05$ & {$[25.4]$} & - & $7.3 / 7$ \\
$R$ & $1.45 \pm 0.03$ & {$[25.27]$} & - & $7.4 / 11$ \\
$I$ & $1.43 \pm 0.06$ & {$[24.3]$} & - & $12.6 / 12$ \\
$J$ & $0.79 \pm 0.15$ & {$[23.6]$} & - & $7.9 / 3$ \\
\hline \multicolumn{5}{c}{ Fits without SN plus fixed $\alpha$} \\
\hline$B$ & {$[1.48]$} & {$[26.1]$} & - & $8.3 / 5$ \\
$V$ & {$[1.48]$} & {$[25.4]$} & - & $14.0 / 8$ \\
$R$ & {$[1.48]$} & {$[25.27]$} & - & $8.4 / 12$ \\
$I$ & {$[1.48]$} & {$[24.3]$} & - & $13.2 / 13$ \\
$J$ & {$[1.48]$} & {$[23.6]$} & - & $28.4 / 4$ \\
\hline \multicolumn{5}{c}{ Fits with SN1998bw plus fixed $\alpha$} \\
\hline$R$ & {$[1.48]$} & {$[25.27]$} & $3.5 \pm 2.0$ & $5.8 / 11$ \\
$I$ & {$[1.48]$} & {$[24.3]$} & $3.4 \pm 1.7$ & $8.8 / 12$ \\
$J$ & {$[1.48]$} & {$[23.6]$} & $0.9 \pm 0.3$ & $3.6 / 3$ \\
\hline
\end{tabular}

${ }^{a}$ Ratio between the SN 1998bw light peak and that of the best-fit SN.

\subsection{GRB 000911 optical-NIR SFDs}

To study the broadband spectral variability of the afterglow, we subtracted the host galaxy spectrum from the observed fluxes in optical and NIR bands at five epochs after the GRB, at which the sampling has the widest spectral coverage. In the cases in which the data in different bands were not simultaneous, the flux density was interpolated to the reference epoch by using the best-fit power-law decay $\alpha=1.48$. Magnitudes were corrected for Galactic reddening and converted into flux densities following the procedure already described.

The simultaneous afterglow SFDs thus obtained, not further corrected for the intrinsic extinction within the host galaxy, are reported in Fig. 6. Despite the uncertainties (due to the rather large errors on the host galaxy flux densities), a spectral steepening with time is suggested. Assuming a spectrum $F(v) \propto v^{-\beta}$ for all epochs, the $V-R$ color at 1.4 days after the GRB implies a spectral slope $\beta=-0.7_{-3.2}^{+3.4}$. A fit to the broad-band optical/NIR SFDs at the following 4 epochs yields $\beta=0.8 \pm 1.3$ (5.03 days after GRB 000911), $\beta=1.1 \pm 0.3$ (10.94 days), $\beta=1.0 \pm 1.2$ (16.90 days) and $\beta=1.9 \pm 0.7$ (25.00 days). The results obtained for the epochs between $\sim 5$ and $\sim 17$ days after the GRB trigger are broadly consistent with those derived by Lazzati et al. (2001). We remark that the huge errors on the value of $\beta$ referring to the first epoch (September 12.74) are due to two effects: the moderately large uncertainties ( $\sim 0.1 \mathrm{mag})$ on the magnitudes and the very narrow $(\sim 0.1 \mathrm{dex})$ SFD baseline.

If we correct the afterglow data for the absorption as derived from the Hyperz best fits of the host galaxy SFD, we obtain for the OT the spectral slopes $\beta=-1.3 \pm 3.3,0.2 \pm 1.3$, $0.6 \pm 0.3,0.4 \pm 1.2$ and $1.3 \pm 0.7$ at the 5 considered epochs. No substantial difference is found among the considered reddening laws (Stb, SMC and LMC; see also Kann 2004).

\section{Discussion}

Our late-time observational campaign on GRB 000911 has allowed us, for the first time, to directly observe and thus extract important information on its host galaxy; this in turn has helped us to better model the shape and evolution of the light curves and SFDs of the GRB 0000911 afterglow in the optical/NIR domain. In the following we discuss the results obtained on each of these three issues.

\subsection{The GRB afterglow}

The temporal decay index of the afterglow of this GRB is consistent with the decline being wavelength-independent. In the first week, the spectral slope $\beta \sim 1$ (assuming no intrinsic absorption local to the host), combined with the average temporal index $\alpha \sim 1.5$, is consistent with a spherical fireball expansion into a constant-density circumburst medium under the hypothesis that the cooling break frequency of the synchrotron radiation, $v_{\mathrm{c}}$, is above the optical range (Sari et al. 1998). This implies an electron energy distribution index $p \sim$ 3 , to be compared with the range $2-2.5$ found for the $p$ values of GRBs studied by Frontera et al. (2000). It should however be remarked that Masetti et al. (2000) suggested that $p>2.6$ for the afterglow of GRB 990705. Unfortunately, the lack of 

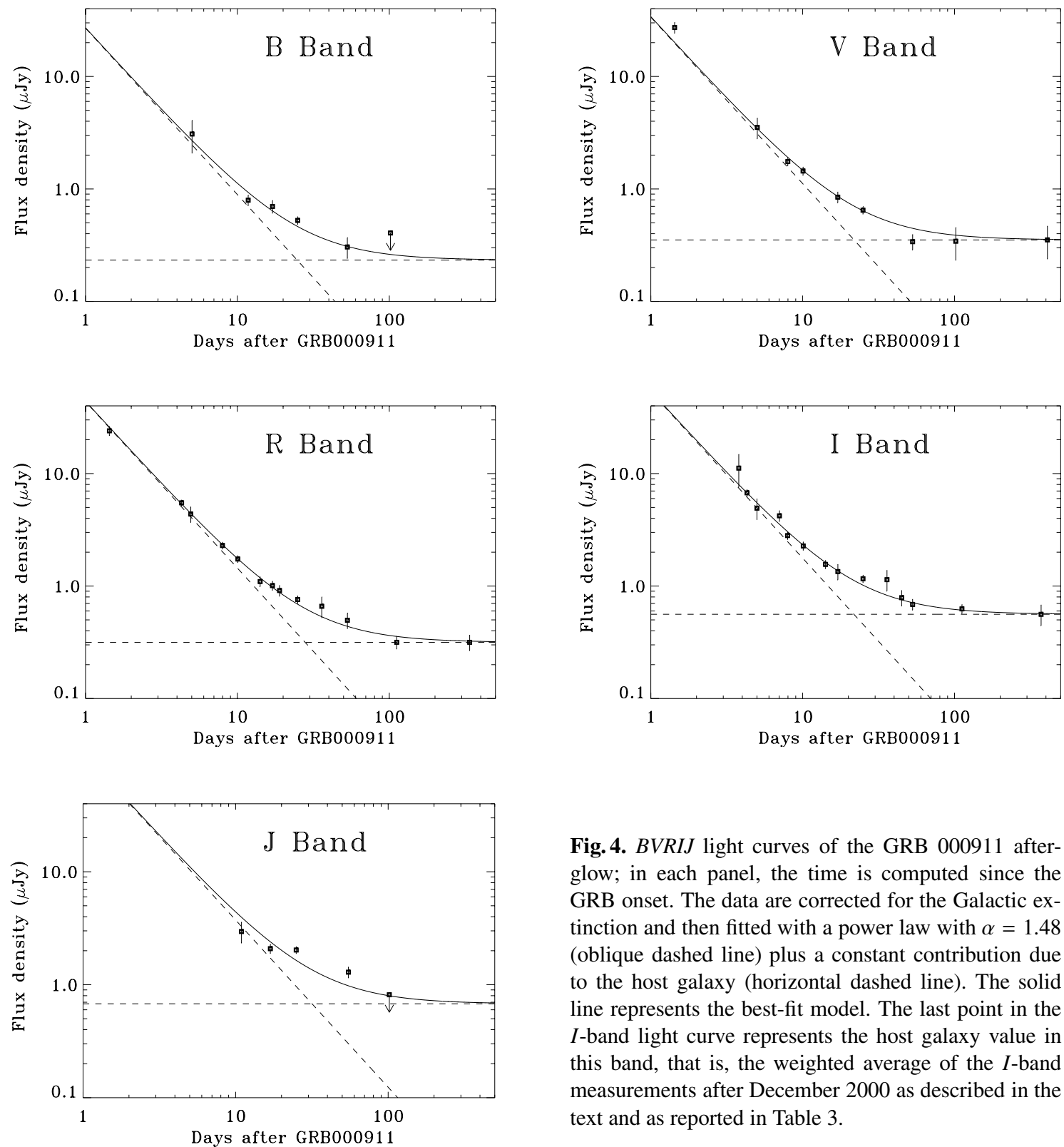

Fig. 4. BVRIJ light curves of the GRB 000911 afterglow; in each panel, the time is computed since the GRB onset. The data are corrected for the Galactic extinction and then fitted with a power law with $\alpha=1.48$ (oblique dashed line) plus a constant contribution due to the host galaxy (horizontal dashed line). The solid line represents the best-fit model. The last point in the $I$-band light curve represents the host galaxy value in this band, that is, the weighted average of the $I$-band measurements after December 2000 as described in the text and as reported in Table 3.

information concerning the high-energy afterglow of GRB 000911 does not allow us to explore in detail more complex models for the fireball expansion. Price et al. (2002), assuming that a light curve break due to collimated emission (as described in Sari et al. 1999) occurred $\sim 1$ day after the GRB 000911 onset, derived $p=1.5$.

The spectral index at 25 days after the GRB, $\beta \sim 1.9$, is again not supportive of a jet geometry of the afterglow emission in the classical collimated fireball scenario (Sari et al. 1999), unless the light curve changes slope at late epochs, possibly implying a more significant supernova contribution. The data are not sufficient to test a change of temporal slope of the afterglow.

A collimated fireball before the jet break (Sari et al. 1999; Rhoads 1999) and an isotropic fireball (Sari et al. 1998) are described in equivalent ways by the standard model, and they cannot be discerned by the temporal and spectral indices. As noted for the case of GRB 020405 (Masetti et al. 2003), if the bump observed in the optical-NIR light curves is due to an emission component independent of the afterglow (i.e., a $\mathrm{SN}$ ), our data cannot rule out a steepening of the afterglow light curve, related to a collimation break, occurring more than $\sim 10$ days after the GRB. According to Sari et al. (1999), this would imply a lower limit to the jet opening angle of $\theta_{0}>10^{\circ}$ assuming a circumburst density $n=1 \mathrm{~cm}^{-3}$ and a GRB isotropic equivalent energy $E_{\text {iso }}=7.8 \times 10^{53} \mathrm{erg}$ (Price et al. 2002).

A "flat $p$ " distribution (i.e. with $p<2$ ), as suggested by Price et al. (2002) for this afterglow, may instead be invoked if local host absorption, as per the Hyperz fit results, is considered. However, in this case the fireball expansion (in the model of Dai \& Cheng 2001) is only compatible with a collimated 

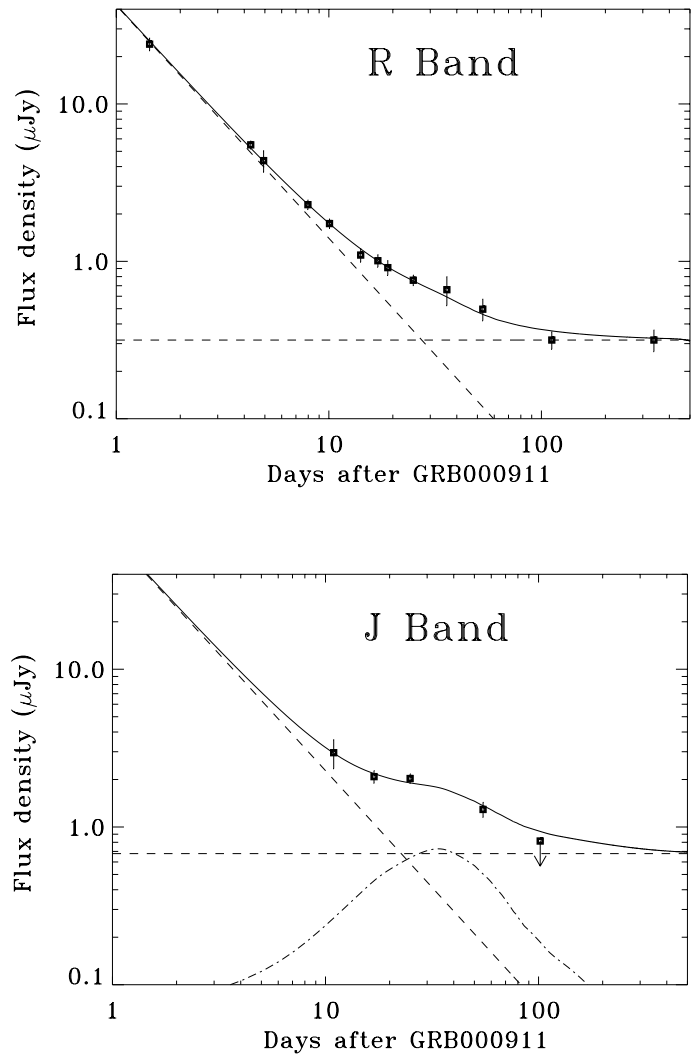

model, assuming a very flat $(p<1)$ electron distribution and $v_{\mathrm{c}}$ below the optical-NIR bands, even accounting for the large errors on $\beta$.

The "inverted" spectral index hinted by the $V R$ data at day 1.44 after the GRB, because of its large uncertainties due to its very short spectral baseline, is in agreement with the fireball model description.

Concerning the very last point in the $I$-band light curve (see Fig. 2 and Table 1), we have no clear explanation for this behaviour, as neither spectral nor color information is available for it, and the temporal sampling is extremely poor. Having excluded any spurious effect as the cause of this variability, for a transient at this redshift we are left with two possibilities: a SN or an Active Galactic Nucleus. The host galaxy optical spectrum (Price et al. 2002), however, does not support the latter explanation. Thus, it could be that a SN, completely unrelated to GRB 000911, exploded in this same galaxy 6 rest-frame months after the GRB. Indeed, given that the host is unresolved in the VLT frames, its star forming regions, and thus any possible transient associated with them, fall within the PSF of the galaxy image. A variable object (possibly a SN) close in the sky to the GRB but unrelated to it was discovered in the field of GRB 020405 (Masetti et al. 2003), and two SNe (2002ap and 2003gd) have recently exploded in the nearby galaxy M 74 (Hirose, reported by Nakano 2002; Evans 2003 ) separated in time by $\sim 1$ year. In any case, given the low significance of the detection and the scanty temporal sampling of the phenomenon, we regard this explanation as tentative at best.

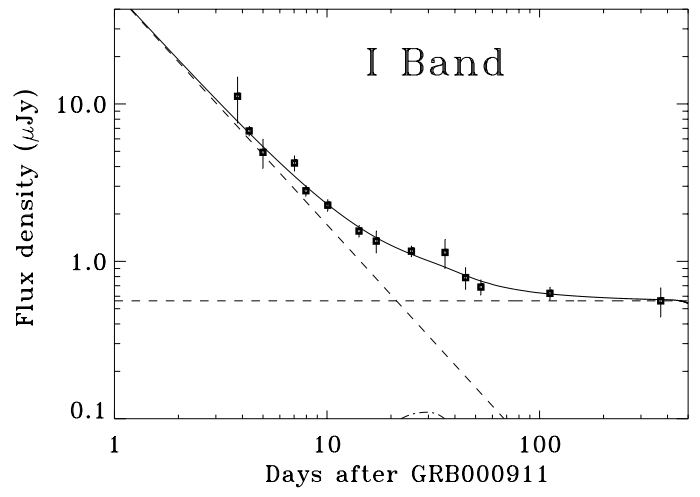

Fig. 5. Same as Fig. 4 for the RIJ light curves of the GRB 000911 afterglow, but with the addition of a $\mathrm{SN1998bw}$ at $z=1.06$ (dot-dashed line; in the $R$-band panel this is not visible) brightened, with respect to the original one, by the factors reported in Table 3. The solid line represents the best-fit model. In these fits the $\mathrm{SN}$ explosion time was assumed to coincide with the GRB start time.

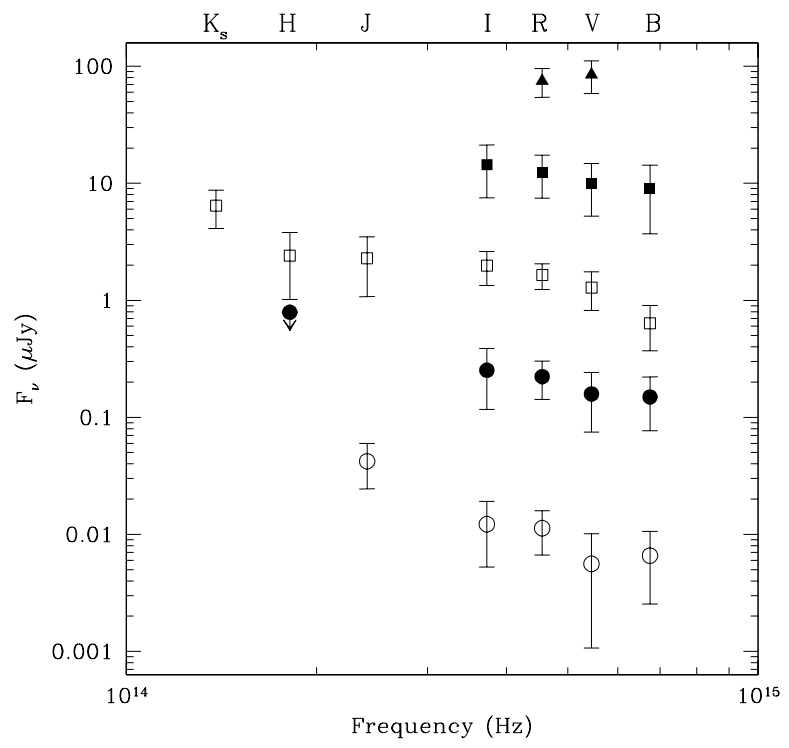

Fig. 6. SFDs of the afterglow of GRB 000911 at 1.44 (September 12.74, filled triangles), 5.03 (September 16.33, filled squares), 10.94 (September 22.24, empty squares), 16.90 (September 28.20, filled circles) and 25.00 (October 6.30, open triangles) days after GRB. The SFDs of September 12.74 and 16.33 are shifted up by 0.5 dex, while those of September 28.20 and October 6.30 are shifted down by 0.5 and $1.5 \mathrm{dex}$, respectively. The data are corrected for Galactic extinction and for the flux of the host galaxy.

\subsection{The associated $S N$}

The results of the fits to the optical and NIR light curves (Table 3) suggest that the presence of a SN underlying the afterglow of GRB 000911 is not required by the optical dataset, 
which is the best sampled, while it is needed at $92 \%$ confidence level to explain the $J$-band data under the assumption that the decay in the NIR range is the same as in the optical. Therefore, it seems that a SN similar to SN1998bw does not contribute significantly to the (observer's frame) optical afterglow of GRB 000911.

As one can see, the SN contribution in the NIR appears to be larger than, although marginally consistent with, that in the optical bands. This marginal discrepancy between the optical and the $J$ light curves might possibly be due in part to the presence of intrinsic reddening local to the host, as pointed out by the Hyperz fit results. Indeed, the observer's frame optical bands correspond to UV and $B$ ranges at $z=1.06$ and are more strongly affected by absorption than the observer's frame $J$ band. A similar discrepancy between rest-frame UV and optical behaviours was found in the case of the possible SN associated with GRB 020405 (Masetti et al. 2003), where the observed $V$-band data (which corresponded to the UV range at the redshift of this GRB, $z=0.69$ ) remained systematically below the best-fit SN curve.

We must however note, as already remarked by Price et al. (2003b), that $\mathrm{SNe} \mathrm{Ib} / \mathrm{c}$ have an intrisic dispersion in the peak luminosity (see Clocchiatti et al. 2000); therefore, in the present case, a SN fainter than $1998 \mathrm{bw}$ could have been associated with GRB 000911. A similar conclusion was reached by Zeh et al. (2004) by considering the $R$-band light curves of 21 GRB afterglows detected up to the end of 2002 and by studying the presence of a possible SN contribution to the total light. Similarly, the SN behaviour in the blue part of the spectrum, especially in the UV range, is still poorly studied and one can not exclude that it may show substantial differences from case to case.

Alternative explanations may also be considered, such as the model developed by Beloborodov (2003) in which the fireball is interacting with a trailing neutron shell; or that in which a SN remnant, located around the GRB progenitor and excited by the GRB itself, cools down on time scales of weeks (Katz 1994; Dermer 2002); or the dust echo model (Esin \& Blandford 2000; Reichart 2001). Ramirez-Ruiz et al. (2001) modeled the GRB 000911 data set of Lazzati et al. (2001) considering the effect of fireball propagation through the progenitor wind. However, the evidence indicating that (at least some) long GRBs are associated with SNe (Galama et al. 1998; Della Valle et al. 2003; Stanek et al. 2003; Hjorth et al. 2003; Malesani et al. 2004) suggests that these alternative models are less viable than the $\mathrm{SN}$ interpretation.

In conclusion, our late-epoch observations have allowed us to determine the host galaxy SFD and therefore to give a more accurate description of the GRB 000911 OT light curves than was previously possible. With these data we could also pinpoint the cause of the OT color evolution stressed by Lazzati et al. (2001): the OT reddening at late epochs is mainly due to the presence of an underlying host galaxy, redder than the afterglow, in the $R$ and $I$ filters, and by a flux enhancement due to an additional (possibly a $\mathrm{SN}$ ) component in the $J$ band. If this phenomenon is indeed produced by the emergence of a Ib/c-type SN component associated with GRB 000911, this is the farthest SN connected with a GRB and, to the best of our knowledge, the farthest core-collapse SN observed up to now.

\subsection{The host}

The temporal extension of our monitoring has allowed us to set some constraints on the host galaxy of GRB 000911. Its spectrum appears to be compatible with that of an irregular galaxy with a moderate amount of intrinsic extinction following a Magellanic-type or a Stb-like reddening law, in agreement with the findings of Lazzati et al. (2001). The host size is very compact: given that the galaxy is unresolved in all of our lateepoch images, we estimated an upper limit of $3.3 \mathrm{kpc}$ for its half-light radius. This prevents us from studying the host morphology with our ground-based observations.

For the host we obtain an absolute $B$-band magnitude $M_{B} \sim$ -18.4 , which compares acceptably well with the values -18.8 to -18.9 obtained for this object by Price et al. (2002) and Le Floc'h et al. (2003). This value is typical of host galaxies of GRBs (see, e.g., Le Floc'h et al. 2003; Christensen et al. 2004; and Sokolov et al. 2001); in particular, the GRB 000911 host falls roughly in the middle of the absolute $B$-band magnitude distribution of the host galaxy sample of Le Floc'h et al. (2003, see their Fig. 6).

In terms of a comparison between the luminosity of the GRB 000911 host galaxy with that of the knee of the galaxies luminosity function, $L^{\star}$ (Schechter 1976), we find (see Table 2) that $L_{\text {host }} \sim 0.07 L^{\star}$ using the luminosity function of Lilly et al. (1995). If we use the luminosity function of Schechter (1976) we get values of $L_{\text {host }}$ which are about twice, in units of $L^{\star}$, those obtained from that of Lilly et al. (1995), as noticed by Gorosabel et al. (2003a). Thus, averaging the two results, we can confidently say that the host of GRB 000911 has $L \sim 0.1 L^{\star}$ and is therefore a subluminous galaxy. This is consistent with the findings of Hogg \& Fruchter (1999) who found that GRB hosts are in general subluminous galaxies.

Using the best-fit templates decribed above and the relation between the rest-frame luminosity at $2800 \AA$ as reported in Kennicutt (1998) we can give an estimate of the SFR in the GRB 000911 host galaxy: from the fits we get a $S F R$ (corrected for the local host absorption) of $\sim 2.7 M_{\odot} \mathrm{yr}^{-1}$ for all cases reported in Table 2. This again is consistent with the $S F R$ value obtained by Price et al. (2002) from the [OII] $\lambda 3727$ line of the host; however, it is slightly different from the value those authors obtained using the $2800 \AA$ flux method. The difference can be attributed to the local absorption we found in our analysis (see also Price et al. 2002; and Lazzati et al. 2001). This further local absorption has been invoked to explain "dark" GRBs (such as GRB 000210; Piro et al. 2002) occurring in host galaxies with low global extinction (Gorosabel et al. 2003a).

Our SFR estimate for the GRB 000911 host, together with a luminosity $L \sim 0.1 L^{\star}$, implies a specific $S F R$ of $\approx 30 M_{\odot} \mathrm{yr}^{-1}\left(L / L^{\star}\right)^{-1}$. This is a very high value: it exceeds by a factor $\sim 3$ the highest specific $S F R$ in the GRB host sample of Christensen et al. (2004), and is an outlier at the high end of the specific $S F R$ distribution of the Hubble Deep Field North (whose galaxies have specific $S F R \mathrm{~s}<25 M_{\odot} \mathrm{yr}^{-1}\left(L / L^{\star}\right)^{-1}$; Christensen et al. 2004). We thus conclude that the host galaxy of GRB 000911 is a very compact, subluminous, slightly reddened, extreme starburst galaxy. 
Concerning the age of the dominant stellar population, when we make a comparison with the sample of Christensen et al. (2004) we see that, according to our best-fit results, the GRB 000911 host falls at the high end of the age distribution. This would suggest that the dominant stellar population is older than the one of the GRB progenitor; this age implies that this main population formed at a redshift $z \sim 1$.3. A similar but more extreme result was found by Levan et al. (2004) for the case of the host galaxy of GRB 030115. A relatively old age for the GRB 000911 host stellar population is mainly inferred by the presence of a discontinuity between the $R$ and $I$ optical bands, which is interpreted by Hyperz as the Balmer break placed around $4000 \AA$ restframe. We however caution that it is difficult to distinguish older populations from dusty environments in high-redshift galaxies with the use of photometric techniques alone (Moustakas et al. 2004).

The host reddening is indeed moderate but within the range of the GRB host samples of Sokolov et al. (2001) and Christensen et al. (2004). No signs of significant intrinsic extinction are however seen in the afterglow SFD; this is possibly because the dust surrounding the GRB may have been destroyed by the initial high energy and UV radiation (Waxman \& Draine 2000; Fruchter et al. 2001). This is one of the few hosts for which NIR data are available (Bloom et al. 1998; Djorgovski et al. 1998; Fruchter et al. 1999a,b; Frail et al. 2002). Its dereddened $(V-H)_{\mathrm{AB}}$ color $^{7}, 1.12 \pm 0.46$, compares well with those of other GRB host galaxies (Fruchter et al. 1999b). Deep imaging of this rather compact host with HST, however, would allow a more detailed study of its morphology and other properties.

\section{Conclusions}

The late-epoch and long-term optical/NIR photometric monitoring of the GRB 000911 OT presented here allowed an indepth analysis of the host galaxy properties and, in turn, an accurate study of the light curves of the GRB afterglow. The host SFD indicates that this is a subluminous extreme starburst compact galaxy with moderate intrinsic extinction, a luminosity $\sim 0.1 L^{\star}$, an age of $\sim 0.5 \mathrm{Gyr}$, a $S F R$ of $\sim 2.7 M_{\odot} \mathrm{yr}^{-1}$ and a specific $S F R$ of $\approx 30 M_{\odot} \mathrm{yr}^{-1}\left(L / L^{\star}\right)^{-1}$. This is the highest specific $S F R$ value for a GRB host inferred from optical/NIR data. The GRB 000911 afterglow seems to follow an adiabatic evolution typical of a synchrotron fireball with electron distribution index $p \sim 3$ and beaming angle $\theta_{0}>10^{\circ}$. The OT light curves show a single power-law temporal decay, modified at late times by light from the host galaxy, and possibly with a deviation at late epochs due to an emerging SN contribution. This component is more apparent in the NIR and could be modeled with a SN similar, in luminosity and overall shape, to SN 1998bw. In this case, it would be both the farthest SN connected with a GRB and the farthest core-collapse SN observed up to now.

Acknowledgements. We are grateful to the anonymous referee for several useful comments which helped us to improve the paper. We thank

\footnotetext{
${ }^{7}$ For a given frequency $v$, the corresponding $\mathrm{AB}$ magnitude is defined (see, e.g., Bolzonella et al. 2000b) as $m_{\mathrm{AB}}=-2.5 \log F(v)-$ 48.60, where $F(v)$ is given in $\mathrm{erg} \mathrm{s}^{-1} \mathrm{~cm}^{-2} \mathrm{~Hz}^{-1}$.
}

the staff astronomers of the ESO (La Silla and Paranal), NOT and TNG observatories. We acknowledge Scott Barthelmy for maintaining the GRB Coordinates Network (GCN) and BACODINE services. We are grateful to $\mathrm{S}$. Covino for having cross-checked his $J$-band data calibration with ours and to D. Lazzati for helpful discussions. We also thank S. Savaglio and E. Rol for useful comments. We acknowledge benefits from collaboration within the EU FP5 Research Training Network "Gamma-Ray Bursts: An Enigma and a Tool". N. Masetti acknowledges support under CRUI "Vigoni" programs 31-2002 and 161-2003. S. Klose acknowledges support from the DAAD under grants $\mathrm{D} / 0237747$ and $\mathrm{D} / 0103745$. This work was partly supported by the Danish Natural Science Reseach Foundation (SNF) and the Carlsberg Foundation. This research has made use of the SIMBAD database, operated at CDS, Strasbourg, France and of NASA's Astrophysics Data System.

\section{References}

Barbon, R., Buondì, V., Cappellaro, E., \& Turatto, M. 1999, A\&AS, 139,531

Beloborodov, A. M. 2003, ApJ, 585, L19

Berger, E., Price, P. A., Frail, D. A., et al. 2000, GCN \#795

Berger, E., Cowie, L. L., Kulkarni, S. R., et al. 2003, ApJ, 588, 99

Bersanelli, M., Bouchet, P., \& Falomo, R. 1991, A\&A, 252, 854

Bloom, J. S., Djorgovski, S. G., Kulkarni, S. R., et al. 1998, ApJ, 508, L21

Bloom, J. S., Kulkarni, S. R., Djorgovski, S. G., et al. 1999, Nature, 401, 453

Bolzonella, M., Miralles, J.-M., \& Pelló, R. 2000a, A\&A, 363, 476

Bolzonella, M., Miralles, J.-M., \& Pelló, R. 2000b, Hyperz v1.1 user's manual, http://webast.ast.obs-mip.fr/hyperz/manual.html

Calzetti, D., Armus, L., Bohlin, R. C., et al. 2000, ApJ, 533, 682

Cappellaro, E., Turatto, M., \& Fernley, J. 1995, IUE-ULDA Access Guide No. 6, Supernovae, ESA SP-1189

Cardelli, J. A., Clayton, G. C., \& Mathis, J. S. 1989, ApJ, 345, 245

Castro-Tirado, A. J., \& Gorosabel, J. 1999, A\&AS, 138, 449

Castro-Tirado, A. J., Zapatero-Osorio, M. R., Gorosabel, J., et al. 1999, ApJ, 511, L85

Christensen, L., Hjorth, J., \& Gorosabel, J. 2004, A\&A, 425, 913

Clocchiatti, A., Phillips, M. M., Suntzeff, N. B., et al. 2000, ApJ, 529, 661

Dai, Z. G., \& Cheng, K. S. 2001, ApJ, 558, L109

Dar, A., \& De Rújula, A. 2003, MNRAS, submitted [arXiv:astro-ph/0308248]

Della Valle, M., Malesani, D., Benetti, S., et al. 2003, A\&A, 406, L33

Dermer, C. D. 2002, ApJ, submitted [arXiv: astro-ph/0211300]

Djorgovski, S. G., Kulkarni, S. R., Bloom, J. S., et al. 1998, ApJ, 508, L17

Djorgovski, S. G., Kulkarni, S. R., Bloom, J. S., et al. 2001, The GRB Host Galaxies and Redshifts, in Gamma-Ray Bursts in the Afterglow Era, ed. F. Frontera, E. Costa, \& J. Hjorth, ESO Astrophysics Symposia (Berlin: Springer Verlag), 218

Esin, A. A., \& Blandford, R. D. 2000, ApJ, 534, L151

Evans, R. 2003, IAU Circ., 8150

Fitzpatrick, E. L. 1986, AJ, 92, 1068

Frail, D. A., Bertoldi, F., Moriarty-Schieven, G., et al. 2002, ApJ, 565, 829

Frontera, F., Amati, L., Costa, E., et al. 2000, ApJS, 127, 59

Fruchter, A. S., Pian, E., Thorsett, S. E., et al. 1999a, ApJ, 516, 683

Fruchter, A. S., Thorsett, S. E., Metzger, M. R., et al. 1999b, ApJ, 519, L13

Fruchter, A. S., Krolik, J. H., \& Rhoads, J. E. 2001, ApJ, 563, 597 
Fukugita, M., Shimasaku, K., \& Ichikawa, T. 1995, PASP, 107, 945

Galama, T. J., Vreeswijk, P. M., van Paradijs, J., et al. 1998, Nature, 395,670

Galama, T. J., Tanvir, N., Vreeswijk, P. M., et al. 2000, ApJ, 536, 185

Gal-Yam, A., Ofek, E. O., \& Shemmer, O. 2002, MNRAS, 332, L73

Garnavich, P. M., Stanek, K. Z., Wyrzykowski, L., et al. 2003, ApJ, 582,924

Gorosabel, J., Castro-Tirado, A. J., Willott, C. J., et al. 1998, A\&A, 335, L5

Gorosabel, J., Fynbo, J. P. U., Hjorth, J., et al. 2002, A\&A, 384, 11

Gorosabel, J., Christensen, L., Hjorth, J., et al. 2003a, A\&A, 400, 127

Gorosabel, J., Klose, S., Christensen, L., et al. 2003b, A\&A, 409, 123

Greiner, J., Klose, S., Salvato, M., et al. 2003, ApJ, 599, 1223

Hamuy, M., Walker, A. R., Suntzeff, N. B., et al. 1992, PASP, 104, 533

Hamuy, M., Suntzeff, N. B., Heathcote, S. R., et al. 1994, PASP, 106, 566

Henden, A. A. 2000, GCN \#800

Hjorth, J., Sollerman, J., Møller, P., et al. 2003, Nature, 423, 847

Hogg, D. W., \& Fruchter, A. S. 1999, ApJ, 520, 54

Horne, K. 1986, PASP, 98, 609

Hurley, K., Cline, T., Mazets, E., \& Golenetskii, S. 2000, GCN \#791

Kann, D. A. 2004, Diploma Thesis, University of Jena

Katz, J. I. 1994, ApJ, 432, L27

Kennicutt, R. C., Jr. 1998, ARA\&A, 36, 189

Klose, S., Stecklum, B., Masetti, N., et al. 2000, ApJ, 545, 271

Lazzati, D., Covino, S., Ghisellini, G., et al. 2001, A\&A, 378, 996

Le Floc'h, E., Duc, P.-A., Mirabel, I. F., et al. 2003, A\&A, 400, 499

Levan, A., Fruchter, A., Rhoads, J., et al. 2004, ApJ, submitted

Lilly, S. J., Tresse, L., Hammer, F., Crampton, D., \& Le Févre, O. 1995, ApJ, 455, 108

MacFadyen, A. I., \& Woosley, S. E. 1999, ApJ, 524, 262

Malesani, D., Tagliaferri, G., Chincarini, G., et al. 2004, ApJ, 609, L5

Masetti, N., Palazzi, E., Pian, E., et al. 2000, A\&A, 354, 473

Masetti, N., Palazzi, E., Pian, E., et al. 2003, A\&A, 404, 465

Miller, G. E., \& Scalo, J. M. 1979, ApJS, 41, 513

Moustakas, L. A., Casertano, S., Conselice, C. J., et al. 2004, ApJ, 600, L131

Nakano, S. 2002, IAU Circ., 7810

Palazzi, E., Pian, E., Masetti, N., et al. 1998, A\&A, 336, L95

Pandey, S. B., Anupama, G. C., Sagar, R., et al. 2003, MNRAS, 340, 375

Patat, F., Cappellaro, E., Danziger, J., et al. 2001, ApJ, 555, 900

Persson, S. E., Murphy, D. C., Krzeminski, W., Roth, M., \& Rieke, M. J. 1998, AJ, 116, 2475
Pian, E., Fruchter, A. S., Bergeron, L., et al. 1998, ApJ, 492, L103

Piro, L., Frail, D. A., Gorosabel, J., et al. 2002, ApJ, 577, 680

Press, W. H., Teukolsky, S. A., Vetterling, W. T., \& Flannery, B. P. 1992, Numerical Recipes (Cambridge: Cambridge Univ. Press)

Prévot, M. L., Lequeux, J., Prévot, L., Maurice, E., \& Rocca-Volmerange, B. 1984, A\&A, 132, 389

Price, P. A., Galama, T. J., Goodrich, R. W., \& Diercks, A. 2000, GCN \#796

Price, P. A., Kulkarni, S. R., Berger, E., et al. 2002, ApJ, 573, 85

Price, P. A., Kulkarni, S. R., Berger, E., et al. 2003a, ApJ, 589, 838

Price, P. A., Kulkarni, S. R., Schmidt, B. P., et al. 2003b, ApJ, 584, 931

Protassov, R., van Dyk, D. A., Connors, A., Kashyap, V. L., \& Siemiginowska, A. 2002, ApJ, 571, 545

Ramirez-Ruiz, E., Dray, L. M., Madau, P., \& Tout, C. A. 2001, MNRAS, 327, 829

Reichart, D. E. 2001, ApJ, 554, 643

Rhoads, J. E. 1999, ApJ, 525, 737

Rhoads J. E., \& Fruchter, A. S. 2001, ApJ, 546, 117

Sari, R., Piran, T., \& Narayan, R. 1998, ApJ, 497, L17

Sari, R., Piran, T., \& Halpern, J. P. 1999, ApJ, 519, L17

Seaton, M. J. 1979, MNRAS, 187, 73

Schechter, P. 1976, ApJ, 203, 297

Schlegel, D. J., Finkbeiner, D. P., \& Davis, M. 1998, ApJ, 500, 525

Smith, I. A., Tilanus, R. P. J., Wijers, R. A. M. J., et al. 2001, A\&A, 380,81

Sokolov, V. V. 2001, Evidence for a Supernova in the $I_{c}$ Band Light Curve of the Optical Transient of GRB 970508, in Gamma-Ray Bursts in the Afterglow Era, ed. F. Frontera, E. Costa, \& J. Hjorth, ESO Astrophysics Symposia (Berlin: Springer Verlag), 136

Sokolov, V. V., Fatkhullin, T. A., Castro-Tirado, A. J., et al. 2001, A\&A, 372, 438

Stanek, K. Z., Matheson, T., Garnavich, P. M., et al. 2003, ApJ, 591, L17

Stetson, P. B. 1987, PASP, 99, 191

Šimon, V., Hudec, R., Pizzichini, G., \& Masetti, N. 2001, A\&A, 377, 450

Tanvir, N. R., Barnard, V. E., Blain, A. W., et al. 2004, MNRAS, 352, 1073

Vietri, M., \& Stella, L. 1998, ApJ, 507, L45

Vreeswijk, P. M., Galama, T. J., Owens, A., et al. 1999, ApJ, 523, 171

Waxman, E., \& Draine, B. T. 2000, ApJ, 537, 796

Zeh, A., Klose, S., \& Hartmann, D. H. 2004, ApJ, 609, 952 